\title{
Top predators and biodiversity: much debate, few data
}

\author{
Fabrizio Sergio $^{1 *}$, lan Newton ${ }^{2}$ and Luigi Marchesi ${ }^{1}$ \\ ${ }^{1}$ Department of Applied Biology, Estación Biológica de Doñana, CSIC, Avda. Mar'a Luisa s/n, Pabellón del Perú, 41013 \\ Seville, Spain; and ${ }^{2}$ Centre for Ecology and Hydrology, Monks Wood, Abbots Ripton, Huntingdon, Cambridgeshire PE28 \\ 2LS, UK
}

\begin{abstract}
Summary
1. In two recent papers, we found support for the hypothesis of an association between the occurrence of top predators and biodiversity. Such results were recently criticized.

2. Cabeza, Arponen \& Van Teeffelen (2008) claim that the proper way to test the investigated relationship was to conduct a regional-level complementarity analysis that pools all predators across all habitat-types. However, such an approach may not help in assessing the performance of predators as surrogates for wider biodiversity, because: (a) management programmes on top predators almost invariably focus on a single species; (b) conservation planning and management often occur within habitats, not only across them; and (c) the required surveys and analyses may often be logistically unfeasible. Re-analysis of our data suggests that a different methodology would not change the results.
\end{abstract}

3. Kéry, Royle \& Schmid (2008) used data from a Swiss volunteers' monitoring scheme to claim that we missed a large number of species in our estimates of avian richness. However, we list 10 reasons why their calculations were inflated, given major differences in surface-coverage, habitatsampling, field effort and in the way that secretive species were treated. Furthermore, our results for birds were replicated in analyses of butterflies and trees, which could not suffer from the same detectability biases.

4. Roth \& Weber (2008) analysed the same Swiss database and found that a group of lower-trophiclevel species (tits) out-performed raptors as indicators of butterfly richness (but not of bird richness). However, their analyses did not control for ecosystem identity, and data on raptor locations were of dubious quality. Furthermore, because tits are major caterpillar predators, the reported findings could have been expected on other grounds.

5. Synthesis and applications. We disagree with Cabeza and colleagues that local studies are of limited importance. Given the paucity of available information, many more local studies are needed to check the generality of the ecological pattern we found. Until further analyses become available, polarized positions for or against top predators as biodiversity indicators are premature. Meanwhile, like any tools, top predators should be employed in conservation decision-making with appropriate caution.

Key-words: indicator species, surrogate species, vertebrate predators

\section{Introduction}

In two recent papers (Sergio, Newton \& Marchesi 2005a; Sergio et al. 2006a), we reported a spatial association between top predators and biodiversity at localities in the Italian Alps. Our findings were criticised by Cabeza, Arponen \& Van Teeffelen (2008; hereafter 'Cabeza'), Kéry, Royle \& Schmid (2008; hereafter 'Kéry'), and Roth \& Weber (2008; hereafter

*Correspondence author. E-mail: fsergio@ebd.csic.es
'Roth'). Space constraints prevent us from dealing fully with these criticisms, but we respond below to the main points in each.

\section{Reply to Cabeza \\ ASSOCIATION BETWEEN TOP PREDATORS AND BIODIVERSITY}

Cabeza claim that, to test an association between top predators and biodiversity, we should have assessed the richness of all 

top predators at a variety of sites across all habitats of our region and tested its congruence with biodiversity. This ambitious approach is challenging, but addresses a different question from the one we posed. It does not detract from our findings for four reasons.

1. Our papers were based on the premise that top predators are over-used in conservation programmes. We therefore posed the question whether such over-focus may deliver broader biodiversity targets. Because nearly all relevant conservation projects deal with one predator at a time (numerous examples in Entwistle \& Dunstone 2000; Caro et al. 2004; Sergio et al. 2008), the most appropriate study design to answer that question is to test the biodiversity-indicator value on a per species basis. Note that most previous tests of the same hypothesis used a similar design (e.g. Caro et al. 2004; Bifolchi \& Lode 2005; Ozaki et al. 2006; Ripple \& Beschta 2006). For example, suppose that a timber company decided to protect a certain amount of forest and impose certain operational restrictions so as to conserve spotted owls Strix occidentalis (as shown in Noon \& McKelvey 1996). We want to know whether such a procedure will benefit spotted owls only, or will help in meeting wider biodiversity targets, or whether the choice of a different species would protect even wider biodiversity. In such common scenarios, measuring the diversity of all vertebrate top predators at these sites would not necessarily answer the question of whether the focus on spotted owls alone was justified.

2. A top predator will usually exert its role in a specific ecosystem (or habitat), rather than over a whole heterogeneous region. To test whether the occupation of a high trophic level implies a link with biodiversity, comparisons should therefore be made within ecosystems, because biodiversity may vary markedly among the ecosystems of a region. For example, in the Alps, golden eagles Aquila chrysaetos L. are top predators in the high-elevation Alpine grassland (Sergio et al. 2006b). If you were to assess the biodiversity indicator role of this species in the Alpine region, you would not pool data from high and low elevation sites. This is because golden eagles do not occupy a top predatory position in low elevation ecosystems and therefore are not expected to reflect low-elevation biodiversity. In agreement with this, if you compare the richness of bird, amphibian and reptile species (recorded through atlas projects, see below) between $100 \mathrm{~km}^{2}$ quadrats occupied or not by the eagles, there is no link between eagles and biodiversity when pooling all regional biota, but a positive association when restricting the comparison to high-elevation species (Fig. 1). In contrast, eagle owls Bubo bubo exert a top predatory role in low elevation ecosystems of the Alps and, as expected, reflect biodiversity within such areas (Sergio, Marchesi \& Pedrini 2004).

3. Given the above rationale of study design, it is clearly more desirable to sample different predators in different study areas so as to make the tests more independent rather than to pseudoreplicate the same test on different species in a smaller area, as proposed by Cabeza.

4. The primary purpose of using surrogate species is to reduce the number of parameters needed to encapsulate a

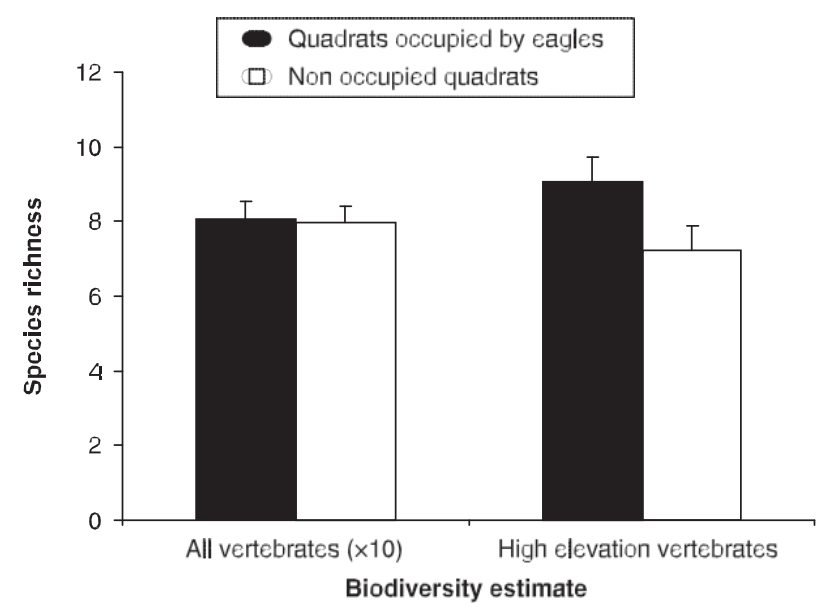

Fig. 1. Mean cumulative number of species of birds, amphibians and reptiles recorded at 25 quadrats of $100 \mathrm{~km}^{2}$ occupied by golden eagles for breeding and at 25 quadrats not occupied by eagles for breeding in the Trento region (central-eastern Italian Alps). Data from Sergio et al. (2006b) and Sergio \& Pedrini (2007).

complex situation (Caro \& O’Doherty 1999). Assessing the distribution of all vertebrate top predators across all habitats of a large region is a formidable task that clearly defies the surrogate purpose itself. We are not aware of any study that has accomplished such a task at the level of detail illustrated in our papers in any other part of the world. Note that all previous intensive tests of the association between top predators and biodiversity focused on one predator species at a time and used sample sizes of 1-21 predator sites (review in Sergio et al. 2008). These figures alone hint at the difficulties in expanding the approach to a wider range of species. If so much field effort had to be employed, then it would be better directed at collecting cheaper, more direct estimates of biodiversity. In sum, the approach proposed by Cabeza may prove interesting, but would be logistically unfeasible and beyond the capability of most funding agencies. Furthermore, even if such an analysis showed an association between top predators and biodiversity, its usefulness elsewhere would be limited to a handful of regions worldwide where predator occurrence is known in sufficient detail for all species over large areas.

The second major criticism of our study design was that the choice of areas sampled was biased towards the occurrence of avian top predators: such focus could have biased the results by missing sites rich in biodiversity but where avian top predators did not occur. This argument also has inherent flaws.

1. We surely accept that some species occupy habitats not included in our survey. However, these habitats are also likely to have their own suite of top predators. We are not aware of any habitat in our study region that lacks top predators, and doubt that such habitats or ecosystems may be common anywhere in the world. At best, for certain microhabitats the top predator could be an invertebrate rather than a bird of prey or mammalian carnivore. The prediction would still be the same: that, whatever the taxonomic identity of the top 
predator, its ecological role causes some link with wider biodiversity.

2. We sampled all the major macro-habitats of the region (woodland, grassland and farmland, collectively accounting for $77 \%$ of our $6300-\mathrm{km}^{2}$ study region), to make the study as representative as possible. Adding other macro-habitat types does not seem to alter the general pattern found, because in other analyses we found similar relationships between top predator occurrence and biodiversity estimates in pre-Alpine lakes (Sergio et al. 2003), low-elevation biota (Sergio et al. 2004) and in high-elevation Alpine grassland (Fig. 1).

3. As stated above, the rationale of a study of this kind is to test whether a top predator will be a reliable biodiversity indicator for its ecosystem alone. To test this notion, given the large amount of fieldwork required, some sort of sampling (of habitats, of predator species, of control surrogate species, of biodiversity estimates) is obviously required, as we cannot record everything. Note that the complementarity analyses proposed by Cabeza also rely heavily on sampling. In fact, some of the complementarity studies cited in Fig. 1 of Cabeza were based on lower sampling rates (number of sample points per unit area) than our study, which means that they could have also missed important, biodiversity-rich sites.

4. At some spatial scale, even the most sophisticated priority-setting algorithms are likely to miss some biodiversityrich sites, because of sampling methodology, stochastic effects (e.g. high biodiversity values only in certain years, or when the site was not sampled) or computational errors. For this reason, some sort of local expert knowledge should always be integrated into local conservation programmes, thus striving for mixed conservation strategies (see Conclusions below).

The third issue raised by Cabeza is that the positive association that we found between raptors and biodiversity may have been caused by a preference of raptorial species for heterogeneous landscapes modified by human action. With such a possibility, they suggest as more desirable the use of species with more specialized habitat requirements. This argument puzzles us for three reasons. (i) As a group, birds of prey are extremely diverse and include species that adapt well to human-modified landscapes as well as more specialist species that fare badly under such conditions (e.g. Mathilde \& Thiollay 1996; Thiollay 1998). The same can be said of most groups of vertebrate top predators. The set of raptor species that we used also included a mix of more and less specialized species. For most predator taxa, it would be easy for an experienced ecologist to select adaptable or specialist species, depending on the aim and setting of a conservation programme. (ii) Biodiversity is not exclusively high in disturbed landscapes, nor in pristine habitats. For example, in certain regions biodiversity may be high in landscapes highly modified by human action, such as the Spanish 'dehesas' of Europe, while in other regions biodiversity may peak in pristine rainforests (e.g. Pain \& Pienkowski 1997; Groombridge \& Jenkins 2002). Therefore, in some regions the association of top predators with disturbed landscapes supposed by Cabeza could even be desirable because the predators would then signal biodiversity- rich habitats. (iii) In all our analyses, the performance of top predators as biodiversity indicators was consistently better than the performance of lower-trophic-level species with specialized ecological requirements. Such results further undermine the assertions of Cabeza.

\section{TOP PREDATORS AS BIODIVERSITY INDICATORS: APPLICATIONS TO PRIORITY-SETTING EXERCISES}

In our papers, we conducted a simulation of a reserve-network selection procedure following the methodology outlined by Kerr (1997), which is included as a reliable case of complementarity analysis by Cabeza et al. (2008, Fig. 1). In this simulation, for each type of location (predator sites, random sites, or sites used by lower-trophic-level species) we (i) selected the site with the highest richness of bird species, and (ii) progressively added sites with sets of species most complementary to those already selected, until all avian species were represented in a hypothetical reserve network. The aim in each case was to find the minimum number of reserves necessary to contain all species encountered during the study. The simulation was repeated separately for each predator, and thus separately within each habitat type. Cabeza argued that we should have pooled all predators and habitats in a single simulation to take into account regional-level complementarity, rather than within-habitat complementarity. They define their proposed simulation as 'systematic' and ours as 'unsystematic'. However, the two approaches are simply different methods, both of them similarly quantitative and systematic, that answer different questions. For example, there are numerous instances in which conservation priorities need to be assessed within habitat-types rather than at a regional level. Thus, forest habitats are often managed separately from other habitats by private companies or by governmental forestry departments. These bodies frequently seek ways to protect or manage biodiversity within the forest habitat by focusing on surrogate species (e.g. Hunter 1999; Lindenmayer, Margules \& Botkin 2000). In this context, top predator species, such as goshawks Accipiter gentilis L. and spotted owls, are used over large areas as management indicator species (Noon \& McKelvey 1996; Caro \& O’Doherty 1999; Ozaki et al. 2006). Clearly, in this sort of scenario, a regional-level complementarity analysis over all habitats would not help the forest manager to prioritize sites.

In addition, we doubt that detailed data on top predator distributions will be frequently available over vast enough areas to conduct such regional-level analyses. The only datasets of this kind that we can think of are atlas data, i.e. coarse-level, presence-absence data usually recorded in large quadrats ( $\geq 100 \mathrm{~km}^{2}$ each). However, we express caution about analyses of top predators based on atlas data for three reasons. (i) Atlas census techniques are often poorly suited to detect top predators. In fact, these groups are already covered independently in some areas by targeted search schemes (e.g. Bijlsma 1993; Thiollay \& Bretagnolle 2005). Owls, in particular, are hard to detect by normal atlas census procedures. (ii) Atlas data do not usually incorporate any measure of site quality 
for the predators, or may not distinguish between breeders and non-breeders. In our analyses, we focused on breeding sites, so as to be sure to sample sites that are really important for the predators. (iii) Conservation programmes and protected areas are often planned and managed at a smaller scale than that usually employed in atlas projects. Other types of analyses would still be necessary in such cases.

Finally, we doubt that a regional-level complementarity analysis would have changed our results for two reasons: (i) our sampled macro-habitats were sufficiently different from one another to avoid excessive overlap in community composition; (ii) there is no reason to expect the communities associated with predator sites to overlap more in species composition across habitats than the communities associated with control sites or lower-trophic-level species. In fact, when we pool all habitats and species together in a single sample, networks based on top predators need 19 protected sites to reach the maximum biodiversity attainable by this group, which is 63 species. The corresponding figures are 20 sites and 52 species for lower-trophic-level species with specialized ecological requirements, 20 sites and 52 species for lowertrophic-level species with unspecialized ecological requirements, and 20 sites and 50 species for random sites. It would take only 10 predator sites (i.e. 50\% less sites) to reach the maximum level of species coverage attained by any set of lower-trophic-level species or random sites. Furthermore, networks planned using lower-trophic-level species failed to represent $24 \%$ of the species richness in the overall sample, against only $7 \%$ for networks planned using top predators. Therefore, taking into account regional-level and cross-habitat complementarity leads to similar results.

\section{Reply to Kéry}

Kéry criticised our estimates of avian richness. They compared them with what they expected on the basis of a sample of quadrats monitored by volunteers in a nearby region of Switzerland, and claimed that we recorded a small, biased portion of the actual species in the community. Below, we explain why we believe that the simulations by Kéry are flawed, and that their criticisms are misguided.

In our study, we assessed the richness of bird species at each sample location (raptor nest or control location) by replicating the same field procedure. Birds were identified by voice recognition during a 10-minute point count, followed by walking slowly for $500 \mathrm{~m}$ in each of the four cardinal directions without stopping, recording all bird species not previously recorded. We further explained that 'each assessment reflected the biodiversity of an area of approximately $1 \mathrm{~km}^{2}$, (Sergio et al. 2006a). To predict the number of species we 'should' have detected in our samples, Kéry used data for 85 quadrats of $1 \mathrm{~km}^{2}$ located in the Swiss Alps, and surveyed by volunteers through irregular routes of $0 \cdot 2-8 \cdot 4 \mathrm{~km}$, single visits of 30 400 min duration, and a cumulated time spent yearly in each quadrat of $1.5-18 \cdot 8 \mathrm{~h}$. To make their data comparable with ours, Kéry statistically re-scaled their survey-routes to a length of $2 \mathrm{~km}$ (i.e. similar to the length of our routes). On the basis of such calculations, Kéry estimated that we missed 79\% of the expected species.

There are numerous reasons why the estimates of species richness recorded by the two survey-designs cannot be directly compared in the simple manner proposed by Kéry.

1. We have serious doubts that the study area employed by Kéry to infer the structure of our avian community is so similar to our Trento study area. Despite their relatively close locations (53-200 km between plots), the two regions belong to different countries, only one of which is in the European Union. This implies major differences in land-use management. For example, subsidies from the Common Agricultural Policy have a major impact on avian taxa throughout most of Europe (Pain \& Pienkowski 1997), but not necessarily in Switzerland. Similarly, numerous subsidy or policy schemes exist in the Swiss Alps but not in the Italian Alps (e.g. the Swiss Agri-environment Scheme, Knop et al. 2006). Furthermore, internal, structural management of many habitats differ between the two countries. For example, Switzerland employed silvicultural methods compatible with the current definitions of 'ecological forestry' about a century before Italy (Piussi 1994), leading to profound differences in the structure and current extent of mature forests in the two countries. Given the above, we doubt that data from one region should be used to predict the species complement in the other, especially when habitat composition is not taken into account (see below).

2. Secondly, when we planned our field study, our main concern was not to find every species present (this is probably never achieved even by the best survey design), but to devise a field procedure that could be exactly replicated at sites occupied or not by raptors, in order not to bias the comparison. For this reason, all field data were collected by the same two researchers working together (L.M. and F.S.), the survey-routes were standardized as described above, and we always sampled a raptor site after a non-raptor site or vice versa, thus preventing biases linked to differential sampling dates or hours. This is a survey design where biases due to observer identity, survey length and timing of data collection are standardized a priori. In contrast, Kéry employed a dataset collected by numerous volunteers with differing skills and field effort. The result is a large, 13-42-fold difference in survey length and duration among quadrats. Under this scenario, we agree with Kéry that 'a posteriori' statistical correction of the estimates must be employed as a 'best of a bad job' option. However, when designing any survey, biases should be curtailed as much as possible a priori rather than exclusively a posteriori through statistics (e.g. Scheiner \& Gurevitch 1993). As Krebs (1998) put it, 'do not get the proverbial statistical cart in front of your ecological horse'. Paradoxically, Kéry assign to us the major sources of bias inherent in their own dataset and that we cautiously avoided a priori. We have nothing against national schemes based on volunteers, as they can generate an impressive amount of useful data. However, we disagree that experimental designs operated consistently by professionals should necessarily incorporate a posteriori correction tools for the bias-problems unavoidably incurred by volunteer-based surveys. 
3. In a similar vein, standardizing survey length across treatments means that some species will inevitably be missed. For example, in 54 cases we detected further bird species after the end of a site sampling session but decided not to include them in order to attain full standardization of the field survey design. In 48 cases this happened at raptor sites, suggesting that our tests were probably conservative. Therefore, we already knew that not all species had been regularly detected, but felt that this was desirable in order to ensure equal survey effort across treatments.

4. In our surveys we walked all transects slowly without stopping, which was another a priori decision designed to standardize survey duration. Volunteer surveys usually rely on passionate birdwatchers, who tend to maximize the number of detected species by spending more time in species-rich sites, or devising routes so as to over-sample sites known beforehand to hold more and rarer species. This is the opposite of what we attempted to do and the two datasets are thus not comparable.

Despite the above differences in methodology, we found strangely high the mean value of 32 bird species per sampled site estimated by Kéry for our study. Based on our experience, we doubt that such high numbers could have been attained at any of our sites, however long our sampling periods. We explain below why we believe that the Kéry numbers were inflated in multiple ways.

5. The database used by Kéry employs quadrats assigned a priori to volunteers. The quadrats can include any types of habitat. This differs fundamentally from our survey design, where quadrats were only chosen in a matched manner within a restricted number of macro-habitats (i.e. those occupied by the raptors). For example, in three of the six habitat types that we sampled (corresponding to the three forest raptors and their associated control sites), all the survey routes were located within extensive forests. By drawing quadrats at random, it is likely that Kéry used mainly heterogeneous quadrats. Their avian communities could thus include species from several habitats, including some never sampled in our study. This would greatly inflate their number of expected species per $\mathrm{km}^{2}$ and may largely explain the reported discrepancy in species richness. Note that our restriction of sampled sites to specific habitats was explicitly planned to control for habitat differences between treatments and to ensure that the biodiversity estimates referred to the macro-habitat in which the target raptor acted as a top predator. For example, Tengmalm's owls Aegolius funereus do not hunt in towns in our region, therefore their associated biodiversity estimates should not incorporate urban biota. Once again, calculations by Kéry reflect the difference between a survey planned a priori for a specific task so as to minimize biases, and a generalized monitoring scheme that cannot incorporate such details.

6. In all our analyses, the indicator species (e.g. one raptor species vs. one lower-trophic-level species) were removed from the estimates of species richness, but not by Kéry.

7. Daily point counts are notoriously poorly suited for censusing certain types of elusive species such as raptors, owls or other low-density, secretive or nocturnal species (e.g. nightjars Caprimulgus europaeus L. or woodcocks Scolopax rusticola
L.). Observations of these species during point counts are occasional and unsystematic. Therefore, all such species were removed from our point counts ( $n=6-9$ species per habitat-type, Table S1, Supplementary material). Such a procedure was taken for granted in our original papers and thus not reported (our omission). All these species were included by Kéry and were likely to be more than 20 in their case because they sampled all habitats. We believe that Kéry and Roth should routinely delete such species from their biodiversity estimates because they cannot be reliably surveyed with the methods they employ.

8. The species richness calculated by Kéry assumes that all species present in a region could occur locally within each quadrat of that region. However, this is not so. Specific valleys or subsectors of a region usually support only a portion of the regional species pool. For example, in Table S1 (Supplementary material) we used regional atlas data and our own local knowledge to calculate for each of our six study areas the maximum number of species that we considered as potentially present in each sampled valley/macro-habitat. The numbers were 32, 33, 33, 43, 45 and 51 species. Note that this is the maximum potential for each habitat, and we would not expect to find all these species in any 1-km² quadrat. Kéry calculated that we should have detected 32 species on average at each site. Clearly, if in some cases the local habitat maximum is already 32-33, the number expected on average at a single site within such habitat could never be 32 (because this assumes that all sites support all species, which is biologically unrealistic). Furthermore, when we totalled all the species that we actually detected in each habitat, we see that we were likely to have missed only 0-3 species per habitat type (Table S1, Supplementary material). These species were common and in all cases were missed in one habitat type but recorded in another. This seems an acceptable level of non-detection and argues against the possibility that there were species that we were never able to identify.

9. We stated that our field design of two perpendicular transects of $1 \mathrm{~km}$ reflected an area of approximately $1-\mathrm{km}^{2}$. The 'approximately' is fundamental because we have no way of knowing the exact surface coverage of each survey. If, for example, we could only detect birds up to $150 \mathrm{~m}$ from each transect, the lower area actually covered would be compatible with the species richness calculated by Kéry. Under this scenario, our data should not be compared with a survey that strives to attain full coverage of a $1-\mathrm{km}^{2}$ surface.

10. To make their data comparable to ours, Kéry statistically re-scaled their species richness to correspond to a route length of $2 \mathrm{~km}$. However, this does not take into account the time spent by each volunteer within each route (see point 4 above). Also, Kéry gives a minimum route length per quadrat of $200 \mathrm{~m}$. We doubt that even the best statistical technique can reliably scale up the species recorded in $200 \mathrm{~m}$ to a length of $2 \mathrm{~km}$. Similarly and unavoidably, statistical models in biology incorporate inherent errors, which are difficult to judge in the case of Kéry because no measure of precision or model performance (e.g. \% explained variance) was given for the estimates in their Fig. 1. 
11. Finally, our analyses on butterflies and trees confirmed the results on birds; these could not be affected by detectability issues because all butterflies were captured and trees are obviously hard to miss. Such results were an even stronger test of the hypothesis because these taxa are less trophically linked to raptors than are birds. Interestingly, Kéry did not comment on this fact.

In conclusion, the surveys used by us and by Kéry reflect different approaches to different tasks: ours was a rapid biodiversity assessment testing a specific hypothesis with focus on specific habitats and on a priori control of field biases, while theirs was a national scheme in heterogeneous sample plots based on volunteers where some biases were unavoidably treated a posteriori. Because of differential surface coverage, habitat sampling and field effort, it is unlikely that the two datasets can be reliably compared in the manner proposed by Kéry. Furthermore, the fundamental issue is not whether some species were missed (some are missed in all surveys and statistical corrections also incorporate prediction errors), but rather whether there is any reason to expect a systematic bias towards recording more species at raptor sites. Kéry cited some potential sources of such bias, but none of them apply to our study. Date, time of day and observer identity did not vary across treatments (see above). Mobbing behaviour of passerines could not be involved because all raptor nests were known beforehand, visited cautiously in order not to disturb their occupants, no playback or stimulation was used, and most of them were nocturnal cavity nesters (i.e. virtually invisible during the day unless explicitly looked for). In the only five cases in which a raptor alarmed conspicuously, point counts were abandoned and re-conducted later in the season. Previous knowledge of a site could not introduce biases because the nests of lower-trophic-level species were also known beforehand and survey design (perpendicular transects towards the four main cardinal directions) precluded concentration on particular species-rich locations. Finally, there is a major, inherent inconsistency in the arguments of Kéry. They state that comparisons of sites within the same region should not be conducted without a proper species detectability analysis, but they draw a comparison across a national boundary that does not incorporate any such detectability analysis.

\section{Reply to Roth}

Roth analysed the same dataset as Kéry and showed that (a) seven raptor species performed quite well as indicators of the richness of birds and vascular plants, but less so for butterflies; (b) a group of lower-trophic-level species (tits) performed just as well, and even better than raptors as indicators of butterflies. According to Kéry and Cabeza, such an analysis is flawed because it does not incorporate complementarity or detectability. On the other hand, we applaud the contribution of new results to the debate, but also express concern for three reasons.

1. We reiterate that the comparison between sites occupied by raptors and by other species should control for habitat or ecosystem differences. When data are filtered from very large areas, you may end up comparing a high-elevation forested site occupied by a hawk with a lowland farmland site occupied by a tit. Does this inform us on whether the presence of a top predator within an ecosystem implies a link with biodiversity? For example, Roth showed that common buzzards Buteo buteo L. are negative indicators of butterfly richness. In the Alps, buzzard density increases steeply with woodland extent (Sergio et al. 2005b), quadrats with buzzards will thus tend to be wooded, while butterfly populations are mostly concentrated in flower-rich grasslands. Taking this reasoning to its extreme, would you compare fish species richness in quadrats with and without golden eagles (which forage only over terrestrial habitats)? A more informative test would have been to compare sites occupied or not by raptors and between sites occupied by raptors or tits in a matched manner, for example by using quadrats within a few $\mathrm{km}$ of each other and with similar habitat characteristics.

2. We have serious concern over the quality of the information on raptors yielded by atlas monitoring schemes (see above), especially at such small-scales $\left(1-\mathrm{km}^{2}\right)$. Roth do not state how the raptors were found and counted. In our experience, atlas data depict raptor distributions extremely poorly compared to systematic targeted surveys. We suspect that quadrat occupation in the Roth dataset was not based on nests. This is problematic for raptors because many of them hunt and display over large areas extending in some species more than $3 \mathrm{~km}$ from the nearest occupied nest. Moreover, many raptors, especially owls, are not especially obvious. In contrast, tits can be much more reliably detected and assigned to a quadrat. Furthermore, some of the sample sizes used by Roth are puzzling. If in such a large area only 33-46 quadrats were occupied by such common species as sparrowhawks Accipiter nisus L. and tawny owls Strix aluco L., the question arises how these occupations were detected. Bias towards areas of easy visitation, near paths or towns, are likely. In turn, these may hold biased biodiversity estimates. Also, many of the so-called nonoccupied locations could actually have been occupied by the target raptor species. In our work, we used all nests in a systematically searched contiguous area to avoid the potential biases associated with finding nests opportunistically.

3. The main finding by Roth is that tits performed better than raptors as butterfly indicators. This is intriguing because breeding tits are renowned as specialist caterpillar predators (Perrins 2008). At best, the choice of tits as control species was unfortunate, because indicator performance should ideally be tested with biodiversity estimates as trophically 'disconnected' as possible from the surrogate species (Bifolchi \& Lode 2005).

\section{Conclusions and ways forward}

If we agree that the potential link between top predators and biodiversity is promoted by an ecological function (i.e. occupying an upper trophic level), it follows: (1) that tests of the hypothesis should be conducted within the ecosystem for which a species acts as a predator; and (2) that this is more 
appropriately done by ad hoc studies rather than through broad-scale atlas data which unavoidably mix different ecosystems and incorporate unknown errors regarding predator location. This is a subtle but fundamental problem that permeates all three commentaries on our original papers. However, we recognize that there will be situations in which conservation will necessarily focus on, say, heterogeneous quadrats (e.g. reserve-networks based on the biodiversity estimates of the Swiss monitoring scheme).

Conservation of top predators continues to promote scientific and emotional debate between proponents and detractors. The question arises: are the two positions really so incompatible? We believe not. Conservation science is a complex, multifaceted discipline. Its applications will necessarily reflect a multitude of approaches, scales of study, and socio-economic or political constraints. Proposing a single type of procedure, be it top predator occurrence or reserve-selection-algorithm, as a panacea for biodiversity conservation is unlikely to promote progress. Rather, we believe that mixed, context-sensitive strategies are an essential prerequisite for successful conservation (see similar arguments in Entwistle \& Dunstone 2000; Carignan \& Villard 2002; Groves 2003). For example, a regional-level complementarity analysis could be used to establish a system of reserves. Within some of them and in the nonprotected matrix, a set of surrogates could be used as management indicator species for sustainable resource use (e.g. forestry). Yet other species could be used as flagships for environmental education or to raise funds to increase the number of reserves subsequently, promote connectivity through corridors, or enlarge existing reserves. Clearly, there is ample scope for using surrogates in numerous applications. Depending on local context, top predators may be reliable tools for some of these tasks. In some cases (e.g. biodiversity indicator role), proper testing should ideally precede the actual application. However, this applies to any surrogate group, not solely top predators. Our study shows that a preliminary assessment of the biodiversity indicator role of a surrogate species could be easily incorporated into the early stages of a conservation programme. An adaptive management approach (e.g. Walters 1986) could further help to ameliorate the utilization of such species once results become available to indicate their potential performance as surrogates.

In this context, we also warn against the negative attitudes shown by some academics regarding the use of surrogate species. For example, Cabeza reviewed studies on indicators of complementarity and warned that most groups gave mixed results, concluding that they would not be reliable surrogates. However, their Fig. 1 shows three groups with positive results in $100 \%$ of $6-11$ independent studies. Other groups gave positive results in $75-80 \%$ of studies. Rather than bemoaning a cumulative lack of evidence, a more constructive approach may be to focus on the fact that some groups do seem to yield promising results. Also, some lack of fit in a minority of cases (i.e. variation) is to be expected in any biological exercise of this type. A more fruitful approach may be to promote the utilization of a potentially promising group in a contextdependent approach but with appropriate caution.
As for top predators, we agree that some studies have shown no association with wider biodiversity. In fact, and contrary to what was stated by Cabeza, in both our papers we expressed caution in using our results as self-standing proof of a general pattern (Sergio et al. 2005a: 192; Sergio et al. 2006a: 1054). However, with only four ad hoc studies published so far on the subject (Caro et al. 2004; Bifolchi \& Lode 2005; Sergio et al. 2005a; Ripple \& Beschta 2006), it would be equally premature to discourage top predator utilization for biodiversity preservation in a noninformed way. Moreover, we are concerned that the current emphasis on methodological issues may discourage future research on what is most currently needed, which is further studies. Ironically, while Cabeza suggest that our papers 're-opened' the debate on top predators and biodiversity, we believe that the debate was never properly opened to begin with. Potential surrogates are being judged without proper assessment.

Ultimately, we believe that top predators will continue to be used in conservation, if only because of the profound fascination that they exert on people, which makes them ideal flagships for education and fund-raising. Like all tools, they 'come with instructions' and will need to be used carefully in a context-sensitive manner. We disagree with Cabeza that single analyses from local regions cannot add much to the debate, because it is only through the accumulation of local studies that the generality of the observed patterns can be properly assessed. Such studies should be supported rather than discouraged through the recommendation of overambitious field-designs (e.g. sampling of all the top predator species of a whole region). We reiterate that the major focus in future should be on the analysis of new data. Such data should: (1) be collected through ad hoc studies specifically planned to test the association between predators and biodiversity; (2) include adequate replicates; and (3) broaden the range of ecosystems, geographical regions and predator taxa over those sampled so far. Such studies could then form the basis of comprehensive meta-analyses.

\section{References}

Bifolchi, A. \& Lode, T. (2005) Efficiency of conservation shortcuts: an investigation with otters as umbrella species. Biological Conservation, 126, 523-527.

Bijlsma, R.G. (1993) Ecologische Atlas Van de Nederlandse Roofvogels. Schuyt, Utrecht, the Netherlands.

Cabeza, M., Arponen, A. \& Van Teeffelen, A. (2008) Top predators: hot or not? A call for systematic assessment of biodiversity surrogates. Journal of Applied Ecology, 45, doi: 10.1111/j.1365-2664.2007.01364.x

Carignan, V. \& Villard, M. (2002) Selecting indicator species to monitor ecological integrity: a review. Environmental Monitoring and Assessment, 78, 45-61.

Caro, T., Engilis, A. Jr, Fitzherbert, E. \& Gardner, T. (2004) Preliminary assessment of the flagship species concept at a small scale. Animal Conservation, 7, 63-70.

Caro, T.M. \& O’Doherty, G. (1999) On the use of surrogate species in conservation biology. Conservation Biology, 13, 805-814.

Entwistle, A. \& Dunstone, N. (2000) Priorities for the Conservation of Mammalian Diversity: Has the Panda Had its Day? Cambridge University Press, Cambridge.

Groombridge, B. \& Jenkins, M.D. (2002) World Atlas of Biodiversity. California University Press, Berkeley, CA, USA.

Groves, C.R. (2003) Drafting a Conservation Blueprint: a Practitioner's Guide to Planning for Biodiversity. Island Press, Washington, USA. 
Hunter, M.L. (1999) Maintaining Biodiversity in Forest Ecosystems. Cambridge University Press, Cambridge.

Kerr, J.T. (1997) Species richness, endemism, and the choice of protected areas for conservation. Conservation Biology, 11, 1094-1100.

Kéry, M., Royle, J.A., Schmid, H. (2008) Importance of sampling design and analysis in animal population studies: a comment on Sergio et al. Journal of Applied Ecology, 45, doi: 10.1111/j.1365-2664.2007.01421.x

Knop, E., Kleijn, D., Herzog, F. \& Schmid, B. (2006) Effectiveness of the Swiss agri-environment scheme in promoting biodiversity. Journal of Applied Ecology, 43, 120-127.

Krebs, C.J. (1998) Ecological Methodology. HarperCollins, New York.

Lindenmayer, D.B., Margules, C.R. \& Botkin, D.B. (2000) Indicators of biodiversity for ecologically sustainable forestry. Conservation Biology, 14, 941-950.

Mathilde, J. \& Thiollay, J.M. (1996) Effects of rain forest disturbance and fragmentation: comparative changes of the raptor community along natura and human-made gradients in French Guyana. Journal of Biogeography, 23, 7-25.

Noon, B.R. \& McKelvey, K.S. (1996) Management of the spotted owl: a case history in conservation biology. Annual Review of Ecology and Systematics, 27, 135-162.

Ozaki, K., Isono, M., Kawahara, T., Ida, S., Kudo, T. \& Fukuyama, K. (2006) A mechanistic approach to evaluation of umbrella species as conservation surrogates. Conservation Biology, 20, 1507-1515.

Pain, D.J. \& Pienkowski, M.W. (1997) Farming and Birds in Europe: the Common Agricultural Policy and its Implications for Bird Conservation. Academic Press, London.

Perrins, C. (2008) Tits and Related Species. Oxford University Press, Oxford.

Piussi, P. (1994) Selvicoltura Generale. UTET, Torino.

Ripple, W.J. \& Beschta, R.L. (2006) Linking a cougar decline, trophic cascade, and catastrophic regime shift in Zion National park. Biological Conservation, 133, 397-408

Roth, T. \& Weber, D. (2008) Top predators as indicators for species richness? Prey species are just as useful. Journal of Applied Ecology, 45, doi: 10.1111/ j.1365-2664.2007.01435.x

Scheiner, S.M. \& Gurevitch, J. (1993) Design and Analysis of Ecological Experiments. Chapman \& Hall, New York.

Sergio, F., Caro, T., Brown, D., Clucas, B., Hunter, J., Ketchum, J., McHugh, K. \& Hiraldo, F. (2008) Top predators as conservation tools: ecological rationale, assumptions and efficacy. Annual Review of Ecology, Evolution and Systematics, 39 (in press).

Sergio, F., Marchesi, L. \& Pedrini, P. (2004) Integrating individual habitat choices and regional distribution of a biodiversity indicator and top predator. Journal of Biogeography, 31, 619-628.

Sergio, F., Newton, I. \& Marchesi, L. (2005a) Top predators and biodiversity. Nature, 236, 192

Sergio, F., Scandolara, C., Marchesi, L., Pedrini, P. \& Penteriani, V. (2005b) Effect of agro-forestry and landscape changes on Common buzzards (Buteo buteo) in the Alps: implications for conservation. Animal Conservation, 7, 1725.
Sergio, F., Newton, I., Marchesi, L. \& Pedrini, P. (2006a) Ecologically justified charisma: preservation of top predators delivers biodiversity conservation. Journal of Applied Ecology, 43, 1049-1055.

Sergio, F., Pedrini, P., Rizzolli, F. \& Marchesi, L. (2006b) Adaptive range selection by golden eagles in a changing landscape: a multiple modelling approach. Biological Conservation, 133, 32-41.

Sergio, F. \& Pedrini, P. (2007) Biodiversity gradients in the Alps: the overriding importance of elevation. Biodiversity and Conservation, 16, 3243-3254.

Sergio, F., Pedrini, P. \& Marchesi, L. (2003) Reconciling the dichotomy between single species and ecosystem conservation: black kites (Milvus migrans) and eutrophication in pre-Alpine lakes. Biological Conservation, 110, 101-111.

Thiollay, J.M. (1998) Distribution patterns and insular biogeography of south Asian raptor communities. Journal of Biogeography, 25, 57-72.

Thiollay, J.M. \& Bretagnolle, V. (2005) Rapaces Nicheurs de France: Distribution, Effectifs et Conservacion. Delachaux et Niestlé, France.

Walters, C. (1986) Adaptive Management of Renewable Resources. MacMillan, New York, USA.

Received 30 December 2007; accepted 11 March 2008

Handling Editor: Simon Thirgood

\section{Supplementary material}

The following supplementary material is available for this article.

Table S1. Potential number of avian species present in each macro-habitat and in each study area sampled by Sergio et al. (2005a, 2006a).

This material is available as part of the online article from: http://www.blackwell-synergy.com/doi/full/10.1111/j.13652664.2008.01484.x

(This link will take you to the article abstract)

Please note: Blackwell Publishing is not responsible for the content or functionality of any supplementary materials supplied by the authors. Any queries (other than missing material) should be directed to the corresponding author for the article. 